\title{
Unequal Distributed Spatial Correlation-based Tree Clustering for Approximate Data Collection
}

\author{
Maiying Shen \\ School of Information Science and Engineering \\ Shandong University \\ Jinan, P. R. China \\ shenmaiying@126.com
}

\author{
Shuo Chen \\ School of Information Science and Engineering \\ Shandong University \\ Jinan, P. R. China \\ bitmouse.micky@gmail.com
}

\begin{abstract}
Dividing the networks into several unequal sizes of clusters and the nodes with similar readings and neighboring geographical location are in the same cluster is an effective way to prolong the WSN lifetime. Most applications in WSNs can tolerate certain accuracy loss of the sensor readings and we can exploit the tradeoff between data accuracy and energy consumption. In this paper, we present an improved protocol called UDSCTC (Unequal Distributed Spatial Correlationbased Tree Clustering for Approximate Data Collection). We modify the radius of node competition depending on the distance of the nodes to the sink node to make close to the sink node of the cluster radius decreases, and realize the compromise of energy-consumption between intra and inter clusters. At the same time, we make the clusters' data forwarded to the sink node by multi-hops like tree architecture in traditional network. The method can enlarge the network area. Simulation result shows that UDSCTC has some improvement in the number of the cluster, the energy consumption, etc.
\end{abstract}

Keywords-Spatial-Temporal; Competitive Radius; DSCC; Multi-hop transmission;Similarity

\section{INTRODUCTION}

Wireless Sensor Networks (WSNs) have drawn more and more people's attention and it can be used in many applications in our real life. The low-cost tiny sensor devices' unattended nature makes the WSNs an available tool to gather data from the physical environment by sensing real-world phenomena. Because of the constrained energy, it is important to design of energy-efficient protocol.

A considerable amount of protocols have been presented. The data collection protocols are different from the traditional clustering because it pays more attention on data content. PAQ [6]exploits the probabilistic model which is based on time series forecasting. Some protocols exploit the spatial-temporal correlations such as the EEDC[5], ASAP[2], DSSC[1], DCglobal[4], etc. In EEDC, a centralized algorithm is used to partition sensor networks into disjoint cliques which have similar readings and a round-bin schedule is used to equal the workload of each clique. Spatial and temporal correlations are all represented in ASAP. DCglobal and DClocal are similar agreement and the biggest difference is in the working pattern that DCglobal is a centralized algorithm that all the work of data processing is done in the sink node but the DClocal is in a distributed pattern that the data procession is done on the forwarding way.

In the paper, we present an improved method to prolong the lifetime of WSN by exploiting the spatial correlation which is called Unequal Distributed Spatial Correlationbased Tree Clustering for Approximate Data Collection (UDSCTC) algorithm. It is achieved on the basic of DSCC and changes the nodes' competition radius which makes the clusters be unequal. Then we schedule the network as tree architecture that the cluster head nodes transmit the gathered readings to the sink node by multi-hop transmission. UDSCTC can divide the network with minimizing reading dissimilarity of nodes in the same cluster and can prolong the network lifetime by make the clusters smaller which are nearer the sink.

The rest of the paper is organized as follows: Section II we describe the network model and the important parameters in the algorithm. Section III we begin to discuss the detail implementation procedure of UDSCTC. Performance evaluation and comparison is presented in section IV. Finally, we will have a conclusion of this paper in the section $\mathrm{V}$.

\section{THE MODEL AND DATA STRUCTURE OF THE NETWORK}

\section{A. System model}

In this paper, we design a WSN that contains $\mathrm{N}$ sensor nodes which deployed randomly in an $\mathrm{L}^{*} \mathrm{~L}$ square area. The set of the node is $\mathrm{S}=\left\{\mathrm{s}_{1}, \mathrm{~s}_{2}, \ldots, \mathrm{s}_{\mathrm{N}}\right\}$ and is the ${ }_{i_{\text {th }}}$ node , $|\mathrm{S}|=\mathrm{N}$. The sink node is located at $(\mathrm{L} / 2, \mathrm{~L} / 2)$.

\section{B. data structure definition}

Definition 1: Competitive Radius. In UDSCTC, we reduce the size of cluster which is near the sink node. Supposing the biggest competitive radius is and a control parameter is to make the radius is in the range of $\left[(1-c) \times R_{s c}, R_{s c}\right]$. The nodes' competition radius is same to the EEUC:

$$
\mathrm{R}_{\mathrm{s}_{\mathrm{i}}}=\left(1-\mathrm{c} * \frac{\mathrm{d}_{\text {max }}-\mathrm{d}\left(\mathrm{s}_{\mathrm{i}}, \mathrm{DS}\right)}{\mathrm{d}_{\text {max }}-\mathrm{d}_{\text {min }}}\right) * \mathrm{R}_{\mathrm{sc}} .
$$


Where $\mathrm{d}_{\max }$ and $\mathrm{d}_{\text {minis }}$ are the maximum and minimum distance between two nodes respectively, $d\left(s_{i}, D S\right)$ is the distance between the normal node and the sink node. The neighboring set of the node is called $\operatorname{NBR}\left(\mathrm{s}_{\mathrm{i}}\right)$.

Definition 2: the similarity between two nodes. We use Manhattan distance which is used in many protocols to measure the dissimilarity between two nodes. The similarity function is:

$$
\mathrm{f}_{\mathrm{md}}\left(\mathrm{s}_{\mathrm{i}}, \mathrm{s}_{\mathrm{j}}\right)=\frac{\sum_{k=1}^{\mathrm{q}}\left|\mathrm{v}_{\mathrm{k}}\left(\mathrm{s}_{\mathrm{i}}\right)-V_{k}^{\prime}\left(\mathrm{s}_{\mathrm{j}}\right)\right|}{\mathrm{q}} .
$$

Where $\mathrm{v}\left(\mathrm{s}_{\mathrm{i}}\right)=\left\{\mathrm{v}_{1}, \mathrm{v}_{2}, \ldots \mathrm{v}_{\mathrm{q}}\right\}$ are the sensor readings series of node. If it satisfies the equation that $\mathrm{f}_{\text {md }}\left(\mathrm{s}_{\mathrm{i}}, \mathrm{s}_{\mathrm{j}}\right) \leq \frac{\varepsilon}{2}$, then we say the two nodes are similar. The set constituted by all nodes which are similar to $s_{i}$ is denoted as

SN $\left(s_{\mathrm{i}}\right), \mathrm{SN}\left(\mathrm{s}_{\mathrm{i}}\right) \in \mathrm{NBR}\left(\mathrm{s}_{\mathrm{i}}\right)$.

Definition 3: Similarity coverage rate . Supposing there are $\mathrm{n}$ nodes in set and $\mathrm{m}$ nodes in set. Then the similarity coverage rate is:

$$
C_{r}\left(\mathrm{~s}_{i}\right)=\frac{\left|S N\left(\mathrm{~s}_{i}\right)\right|}{\left|S N\left(\mathrm{~s}_{j}\right)\right|}=\frac{m}{n} .
$$

Definition 4: Similarity difference rate . It is computed by the similarity of the nodes in

$$
S_{r}\left(\mathrm{~s}_{i}\right)=\frac{\varepsilon-\frac{\sum_{\mathrm{s}_{j} \in S N\left(\mathrm{~s}_{i}\right)} f m d\left(\mathrm{~s}_{i}, \mathrm{~s}_{j}\right)}{m}}{\varepsilon} .
$$

Definition 5: Relative energy level. It is the comparison between the remaining energy of the node and the average energy of neighboring set.

$$
E_{R}\left(\mathrm{~s}_{i}\right)=\frac{E\left(\mathrm{~s}_{i}\right) \times(\mathrm{n}+1)}{E\left(\mathrm{~s}_{i}\right)+\sum_{s_{j} \in N B R\left(\mathrm{~s}_{i}\right)} \mathrm{E}\left(\mathrm{s}_{j}\right)} .
$$

\section{THE UDSCTC ALGORITHM}

The UDSCTC protocol is run by round. In each round time, it can be divided into three stages: the clusters formation, the stably data transmission and the cluster maintenance. In cluster formation stage, the sink node broadcasts the clustering-command message. The node can gain the maximization competitive radius and the userdefined error-tolerance threshold $\varepsilon$. Then each node broadcasts a Hello message (H-msg) which contains the residual energy and the recent data series within its own radius range. It also receives some $\mathrm{H}-\mathrm{msg}$ messages from the neighboring nodes.

\section{A. The selection of the cluster heads}

After collecting all the data within the competitive radius, the node will compute the Similarity coverage rate , Similarity difference rate and Relative energy level .If it meets the following two inequalities then it can be selected as the cluster head candidate.

$$
\left\{\begin{array}{c}
\mathrm{E}_{\mathrm{R}}\left(\mathrm{s}_{\mathrm{i}}\right)>1 \\
\mathrm{p}\left(\mathrm{s}_{\mathrm{i}}\right)=\alpha \times \mathrm{C}_{\mathrm{r}}\left(\mathrm{s}_{\mathrm{i}}\right)+(1-\alpha) \times S_{r}\left(\mathrm{~s}_{\mathrm{i}}\right) \geq \delta .
\end{array}\right.
$$

The in inequality (10) is a parameter which can adjust the relative importance of and . is used to control the number of the $\mathrm{CHC}$ nodes.

After being selected as the $\mathrm{CHC}$ node, it broadcasts a competition message (C_msg) which contains the $\mathrm{CHC}$ node's ID, the size of the in the range of $\mathrm{R}(\mathrm{)})$ to compete to be the final cluster heads. In this context, we define that the node defeats node when meeting the following items: they are neighboring nodes; more than half nodes of are in the set of and the size of is bigger than that of the ; is less than and the distance of two nodes is the smaller one of the two nodes' competitive radius.

$\mathbb{R}_{\text {orerlapp }}$, is the overlapping area of two nodes. In Fig.1, the overlapping area is minimized when node is on the circle of .If it meets then our prior assumption is right. It is proved as followed:

$$
\begin{aligned}
& \text { Because , then } \\
& \beta>\partial, \beta+2 \partial=180^{\circ}, \beta>60^{0}, \partial<60^{\circ} . \\
& R_{s_{j}}=x \times R_{s_{i}} \text {, where } \mathrm{x}>1 ; 2 R_{s_{i}}>R_{s_{j}} \text {, then } \mathrm{x}<2 \\
& A_{\text {overlapping }}=\frac{2 \partial \times \pi \times R_{s_{j}}^{2}+2 \beta \times \pi \times R_{s_{i}}^{2}}{360}-\frac{R_{s_{i}}^{2} \times \sin \beta}{2} \\
& \text { The } A_{\text {overlapping }} \text { is minimize when } \mathrm{x}=1, \\
& A_{\text {overlapping }}=R_{s_{i}}^{2} \times\left[\frac{\pi}{180}\left(\partial \times \mathrm{x}^{2}+\beta\right)-\frac{\sin \beta}{2}\right] \\
& \quad=\mathrm{R}_{\mathrm{s}_{\mathrm{i}}}^{2} *\left[\frac{\pi}{180}(\alpha+\beta)-\frac{1}{2} * \sin \beta\right] \\
& \quad=R_{s_{i}}^{2} \times\left[\frac{\pi}{180} \times 2 \times 60-\frac{\sin 60}{2}\right]=1.66>0.5
\end{aligned}
$$




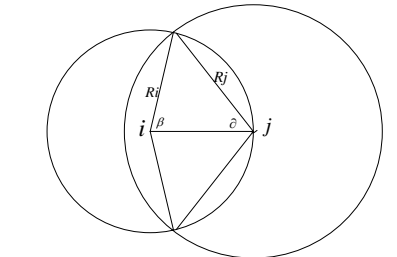

Figure 1. Competition of $\mathrm{CHC}$ nodes.

\section{B. Formation of clusters}

After the $\mathrm{CH}$ nodes being selected, the non- $\mathrm{CH}$ nodes begin to join the appropriate clusters according to the relative distance and the level of similarity by computing the attraction score of the $\mathrm{CH}$ nodes in their. The attraction score of the $\mathrm{CH}$ node is defined by the Eq.(7):

$$
G_{s_{i}}\left(\mathrm{~S}_{C H}\right)=\frac{R_{s_{i}}-\operatorname{dist}\left(\mathrm{s}_{i}, \mathrm{~s}_{C H}\right)}{R_{s_{i}}}+\frac{\varepsilon-f m d\left(\mathrm{~s}_{i}, \mathrm{~s}_{C H}\right)}{\varepsilon}+\gamma .
$$

Where is a similarity importance factor, the distance between the node and the optional $\mathrm{CH}$ node. The non- $\mathrm{CH}$ nodes select the $\mathrm{CH}$ with highest score of the attraction and join the cluster by sending a join message ( $\mathrm{J}$ msg).

\section{Stable data transmission phase}

In DSCC, the clusters directly communicate with the sink node. In this paper, we present a new method to divide network into some layers according to the distance between the $\mathrm{CH}$ nodes and the sink node, the data can be forwarded by the $\mathrm{CH}$ nodes in different layers.

The square sensing area can be seen as a circular area and we divide it into $M$ layers. In order to realize the communication between two adjacent layers, we design the distance of two cutting line of two layers to be . When the sink node is in the center of the sensing area, the value of $\mathrm{M}$ is $\mathrm{M}=\frac{\mathrm{L} / 2}{\mathrm{R}_{\mathrm{sc}} / 2}=\frac{\mathrm{L}}{\mathrm{R}_{\mathrm{sc}}}$.

\section{Maintenance of the clusters}

In order to improve the quality of the clusters, it also has a maintenance process of clusters in UDSCTC. The clusters have the self-adjusting ability to partition the cluster into some smaller clusters when more than one cluster members report seriously different readings. When the energy of the $\mathrm{CH}$ is low or the number of the clusters is beyond a threshold, such as the , the cluster maintenance will be hold.

\section{EVALUATION}

In this section we will compare the UDSCTC protocol with the typical clustering algorithm such as the DSCC, ASAP, EEDC by means of MATLAB. We pay more attention on the following parameters: (1) the number of the clusters; (2) the number of the forced $\mathrm{CH}$ nodes which are the "uncovered"; (3) the standard variance of the size of the clusters, it can be computed by the Eq.(8); (4) the energy consumed in clustering phase; (5) the residual average energy of the $\mathrm{CH}$ nodes; (6) the average similarity of the clusters , it can be gained by the Eq.(9).

$$
\sigma_{c s}=\sqrt{\frac{\sum_{C_{i} \in C}\left(\left|C_{i}\right|-\overline{C_{*}}\right)^{2}}{|C|}} .
$$

$$
V_{d i s}=\frac{\sum_{C_{i} \in C} \frac{\sum_{C_{m} \in C_{i}} f m d\left(\mathrm{C}_{i} \cdot \mathrm{ch}, \mathrm{C}_{i} \cdot \mathrm{cm}\right)}{\left|C_{i}\right|-1}}{C} .
$$

Where the set of the clusters, is the ith cluster and are the $\mathrm{CH}$ node and the member of the ith cluster respectively.

\section{A. Simulation initialization}

As before, we assume $\mathrm{L}=100 \mathrm{~m}$ and $\mathrm{N}$ sensor nodes are randomly deployed in the $100 * 100$ square area. The initial energy of each node is in the range of [4.0,5.0]J. Supposing there are 20 event generators in the sensing area, the data is the temperature within the range of which are chosen from the Intel Lab data set .The sensor node reading is related to the distance to the events and can be described as:

$$
\operatorname{reading}_{\mathrm{s}}(\mathrm{t})=\sum_{i=1}^{20}\left(\frac{\sqrt{\operatorname{dist}\left(\mathrm{s}, \mathrm{e}_{\mathrm{i}}\right)}}{\sum_{j=1}^{20} \sqrt{\operatorname{dist}\left(\mathrm{s}, \mathrm{e}_{\mathrm{j}}\right)}}\right) * \mathrm{e}_{\mathrm{i}}(\mathrm{t})
$$

In UDSCTC, we make the network last for 4000 time units and the buffer of the sensor node can store $\mathrm{q}$ data. It collects the data once per 4 time units and run the cluster maintenance per 40 time units. When the number of incompetent clusters is larger than the threshold, sink node will broadcasts a message to re-clustering the network. The data packet has 1000 bits data and control packet is 100 bits.

\section{B. Analysis of the parameters in the system}

In this paper, we present some parameters, such as the is used to control the number of the nodes to be compared. We just discuss the effect on the dissimilarity of nodes in UDSCTC and we can gain it from Fig.2.

We can see that when the $\mathrm{R}_{s c}$ is $20 \mathrm{~m}$, the number of the clusters and the dissimilarity of the clusters is approximate. Then we will design the $R_{s c}$ to be $20 \mathrm{~m}$ in the following evaluation and the advanced network which is formed by the $\mathrm{CH}$ nodes can be divided into 5 layers. 


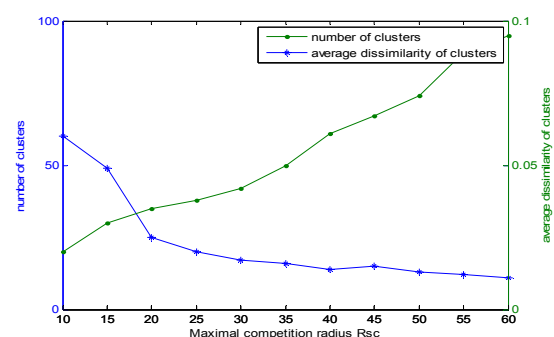

Figure 2. Effect of Fig on UDSCTC

is the threshold of error-tolerance and is used in the selecting of the $\mathrm{CHC}$ nodes. In this section, we also make the $\mathrm{N}$ be 300 and is in the range of $[0.5,0.95]$ which are same in DSCC. From the Fig.3 (a) and Fig.3(b) we can learn the number of clusters and the forced clusters decrease as increases, because the nodes with large dissimilarity may be grouped into the same cluster and the size of the cluster becomes larger. But the number of clusters in UDSCTC is larger than the DSCC, because the average radius of clusters is smaller in UDSCTC. The number of forced clusters is smaller. In Fig.3(c), it describes the residual energy level of $\mathrm{CHs}$ in different algorithm with the changing of , we can see that the residual energy level is higher in UDSCTC than other algorithm. It is the result of adopting the tree architecture and unequal size of clusters.
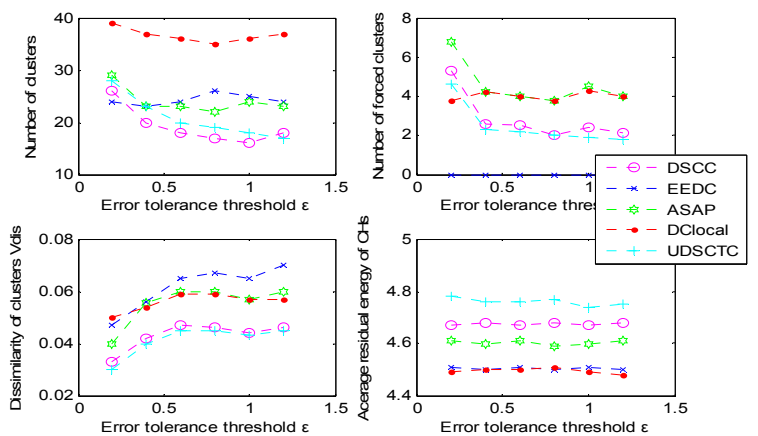

Figure 3. Effect of error-tolerance threshold

is used to control the number of the $\mathrm{CHC}$ nodes. The number of $\mathrm{CHC}$ nodes will be too small to achieve the effect of clustering when the value of is large. is a importance factor to adjust the importance of and in calculating the probability of competing to be $\mathrm{CHC}$ nodes. We can seen from the Fig.4 that when increases, the weight of similarity reduces then the number of clusters will reduce because the nodes in the same cluster have large dissimilarity in the same cluster. When the value of becomes larger, the nodes that have large similarity can be divided into the same cluster and the number of cluster will increase.

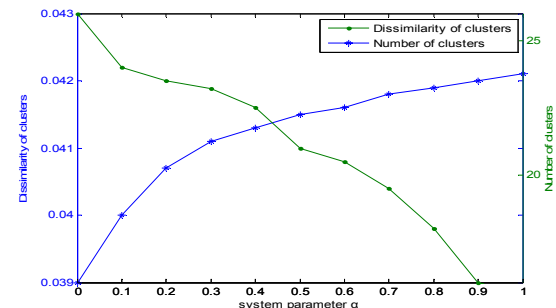

Figure 4. Effect of $\mathbb{F}$ on the similarity and number of clusters

is used to calculate the attraction of the $\mathrm{CH}$ nodes to the normal ones. Its effect is to adjust the weight of the similarity in computing the value of the attraction. It can not affect the number of the $\mathrm{CH}$ nodes but has an opposite effect on the dissimilarity of nodes in the same cluster. The effect can be gained from the Fig. 5 .

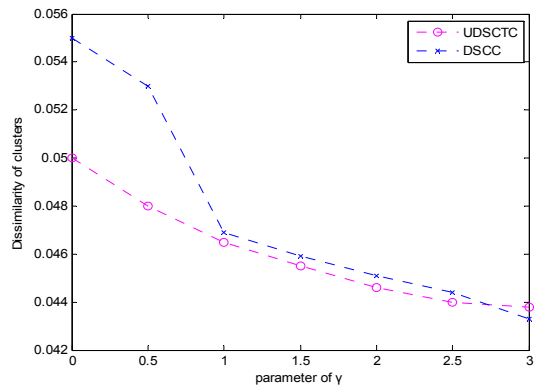

Figure 5. Effect of $\mathbf{E}$ on dissimilarity of clusters

\section{CONCLUSION}

In this paper, we present an improved algorithm called UDSCTC to prolong the lifetime of the WSN. We partition the network into several unequal clusters according to the distance to the sink nod. We also partition the network into several layers and the $\mathrm{CH}$ nodes belongs to lower layer can forward the data to the upper one like tree architecture in traditional wireless networks. In the last, we use MATLAB to evaluate the algorithm and we can see from the result that UDSCTC has large improvement in the energy saving and reducing of the number of the clusters. It can prolong the WSN network effectively. The next work we should is to add the temporal correlation of the readings to the algorithm, and add better linear model to predict the readings of the unsampled nodes.

\section{REFERENCES}

[1] Liu Z, Xing W, Zeng B, et al. Distributed spatial correlation-based clustering for approximate data collection in WSNs[C]//Advanced Information Networking and Applications (AINA), 2013 IEEE 27th International Conference on. IEEE, 2013: 56-63.

[2] Gedik B, Liu L, Yu P S. ASAP: an adaptive sampling approach to data collection in sensor networks[J]. Parallel and Distributed Systems, IEEE Transactions on, 2007, 18(12): 1766-1783.

[3] Hung C C, Peng W C, Lee W C. Energy-aware set-covering approaches for approximate data collection in wireless sensor networks[J]. Knowledge and Data Engineering, IEEE Transactions on, 2012, 24(11): 1993-2007.

[4] Peng C C H W C, Tsai Y S H, Lee W C. Exploiting spatial and data 
correlations for approximate data collection in wireless sensor networks[J]. Knowledge Discovery from Sensor Data (Sensor-KDD 2008), 2008: 111

[5] Liu C, Wu K, Pei J. An energy-efficient data collection framework for wireless sensor networks by exploiting spatiotemporal correlation[J]. Parallel and Distributed Systems, IEEE Transactions on, 2007, 18(7): 1010-1023.

[6] Tulone D, Madden S. PAQ: Time series forecasting for approximate query answering in sensor networks[M]//Wireless Sensor Networks.
Springer Berlin Heidelberg, 2006: 21-37.

[7] Chu D, Deshpande A, Hellerstein J M, et al. Approximate data collection in sensor networks using probabilistic models[C]//Data Engineering, 2006. ICDE'06. Proceedings of the 22nd International Conference on. IEEE, 2006: 48-48.

[8] Ye W, Heidemann J, Estrin D. An energy-efficient MAC protocol for wireless sensor networks[C]//INFOCOM 2002. Twenty-First Annual Joint Conference of the IEEE Computer and Communications Societies. Proceedings. IEEE. IEEE, 2002, 3: 1567-1576. 